\title{
Development of a Point-of-Care Platform for Plant Health Assessment: A Microfluidic Approach ${ }^{\dagger}$
}

\author{
Eduardo J. S. Brás 1,2,*, Ana Margarida Fortes ${ }^{3}$, Virginia Chu 1, Pedro Fernandes ${ }^{2,4}$ and \\ João Pedro Conde ${ }^{1,4}$
}

1 Instituto de Engenharia de Sistemas e Computadores, Microsistemas e Nanotecnologias (INESC MN) and IN, Institute of Nanoscience and Nanotechnology, 1000-029 Lisbon, Portugal; vchu@inesc-mn.pt (V.C.); joao.conde@tecnico.ulisboa.pt (J.P.C.)

2 IBB - Institute for Bioengineering and Biosciences, Instituto Superior Técnico, Universidade de Lisboa, 1049-001 Lisbon, Portugal; pedro.fernandes@ist.utl.pt

3 Faculdade de Ciências de Lisboa, Universidade de Lisboa, BioISI, Campo Grande, 1749-016 Lisboa, Portugal; amfortes@fc.ul.pt

4 Department of Bioengineering, Instituto Superior Técnico, Universidade de Lisboa, 1049-001 Lisboa, Portugal

* Correspondence: ebras@inesc-mn.pt; Tel.: +351-964-754-304

† Presented at the Eurosensors 2018 Conference, Graz, Austria, 9-12 September 2018.

Published: 13 December 2018

\begin{abstract}
Azelaic Acid (AzA) is a signaling molecule that plays a role in plant immune response when these are infected with pathogens, such as Botryitis cinerea, making its early detection in the field critical in the monitoring and prevention of large spread infection in crops. In this work, a microfluidic platform for the rapid detection of AzA in grapes is presented. AzA detection is achieved via an enzyme-enabled colorimetric reaction performed in a microfluidic chip coupled to a thin-film silicon photodiode. The detection of $\mathrm{nM}$ concentrations of AzA in buffer was achieved and initial results for AzA measurements in artificially spiked grape juice show potential for detection in realistic sample conditions.
\end{abstract}

Keywords: azelaic acid; microfluidics; SAR; point-of-care

\section{Introduction}

In a world of increasing antibiotic and antifungal resistance, there is a growing need for rapid and portable infection detection systems. In the wine industry, a serious problem is crop infection by Botrytis cinerea, a common pathogen associated with grapes and vine trees. This necrotrophic pathogen also affects other fruits such as strawberry, orange, tomato and mango. When this fungus infects the plant, an immune response is activated, which is known as SAR-Systemic Acquired Resistance. Within the SAR pathway, a small organic acid, Azelaic Acid (AzA), is produced which acts as a signaling molecule to trigger the immune response, making it a prime candidate for early detection of disease [1]. In order to detect this small acid, we will take advantage of its inhibitory effect over Tyrosinase, which is an oxidase present in several species, including humans, where it participates in the synthesis of melanin [2]. Tyrosinase catalizes oxidation of phenols such as Tyrosine and Catechol to quinones. However, AzA has been reported to be able to competitively inhibit its activity by lodging itself in its active center [2].

In this work, we propose a microfluidic system coupled to an a-Si:H photodiode capable of detecting the presence of AzA colorimetrically in buffer solutions and also in artificially spiked samples of grape juice through the use of Tyrosinase immobilized on microbeads. 


\section{Materials and Methods}

\subsection{Materials and Reagents}

All stock solutions were prepared in potassium phosphate buffer at $\mathrm{pH}$ 6.5, prepared using Mili$\mathrm{Q}$ water. Tyrosinase (from mushroom) stock solution was prepared as $2 \mathrm{~g} / \mathrm{L}$ of enzyme as indicated by the supplier, while Tyrosine and MBTH stock solutions were prepared at concentrations of $2 \mathrm{mM}$ and $100 \mathrm{mM}$, respectively. AzA stock solutions were prepared at a concentration of $1 \mathrm{mM}$. All the aforementioned reagents were acquired from Sigma-Aldrich (Sintra, Portugal). For the spiked samples, ripe Red Globe grapes were purchased at the local market and washed with Mili-Q water.

\subsection{Microfabrication of a-Si:H Photodiodes}

The a-Si:H photodiodes correspond to a vertical p-i-n junction, deposited through radio frequency plasma enhanced chemical vapor deposition (rf-PECVD). All layers were patterned using a DirectWrite Lithography equipment (DWL ii Heidelberg Instruments). For a more detailed description of this process, previous work from our group can be consulted [3].

\subsection{Microfluidic Device Fabrication}

The microfluidic devices are similar to the ones used by Pinto et al. [4] and were fabricated through similar soft lithography techniques. This device consists of two layers, the first being $100 \mu \mathrm{m}$ high, where the microbeads will be packed, and a second region, $20 \mu \mathrm{m}$ high in order to trap the aforementioned beads. The microbeads are packed into the channel by inserting a pipette tip containing a suspension of beads in buffer, into the inlet, and applying negative pressure using a syringe pump at a flow rate of $15 \mu \mathrm{L} / \mathrm{min}$.

\section{Results and Discussion}

The microfluidic system designed to detect AzA consists of a $100 \mu \mathrm{m}$ high PDMS (Polydimethylsiloxane) microfluidic column coupled to a thin-film silicon a-Si:H photodiode. The $200 \times 200 \mu \mathrm{m}$ photodiodes used for the absorbance measurements $(\lambda=480 \mathrm{~nm})$ are aligned with the column as seen in Figure 1A. These columns were then packed with $50 \mu \mathrm{m}$ diameter $\mathrm{SiO}_{2}$ beads functionalized with APTES ((3-Aminopropyl) triethoxysilane), resulting in a positively charged bead surface with a primary amine. These beads served the double purpose of being used to immobilize the enzyme Tyrosinase and also to capture the colored reaction product. As seen in Figure 1B, a mixture of the sample and a known amount of both Tyrosine and MBTH (3-Methyl-2-benzothiazolinone hydrazine) are injected into the channel, and in the case of a non-contaminated sample, the enzyme will oxidize the tyrosine and produce a colored pigment that will deposit on the beads; in a contaminated sample, the AzA will inhibit the enzymatic reaction thus resulting in a reduced color intensity.

Previous work in the literature have reported a spike in AzA after pathogen infection [5], and Jung et al. [1] reported concentrations in the $\mu \mathrm{M}$ range. In this work, we aimed to detect concentrations lower than this in order to take into account any sample pre-treatment steps that could be necessary, so far achieving detection levels in the 50-100 nM range in buffer as seen in Figure 2.

Once the microfluidic AzA detection operating conditions have been established, the next step was to try to detect the presence of AzA in a more realistic situation. Due to the fact that grapes have high quantities of phenols in both their pulp and their skin, some degree of sample pre-treatment was necessary, otherwise the free phenols in the grape juice will also react in a similar fashion to the tyrosine used as a substrate. After grinding the grapes using a mortar and pestle, the pulp was centrifuged at $2000 \mathrm{G}$ for $15 \mathrm{~min}$. The resulting supernatant was then retrieved, spiked with $1 \mu \mathrm{M}$ of AzA and then diluted according to the desired experimental conditions.

The results presented in Figure 3 demonstrate that the presence of AzA in spiked samples can be clearly distinguished from the negative control samples. This proof of the system performance motivates further improvements towards detection in real case scenarios. 


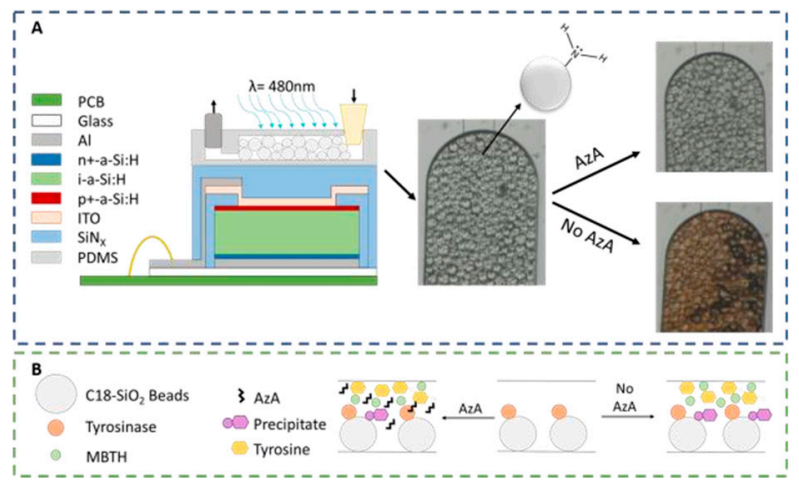

Figure 1. Experimental setup used for the detection of AzA. (A) a-Si:H photodiodes (schematic not to scale) are aligned with a PDMS microcolumn packed with APTESfunctionalized $\mathrm{SiO}_{2}$ beads. The micrographs on the right side of the figure illustrate the optical difference between a contaminated and non-contaminated sample; (B) Representation of the reaction mechanism used as a chemical transducer for AzA detection. In a first step, tyrosine is oxidized to a quinone which then reacts with MBTH to produce a colored pigment which is captured by the beads. AzA acts as an enzymatic inhibitor.
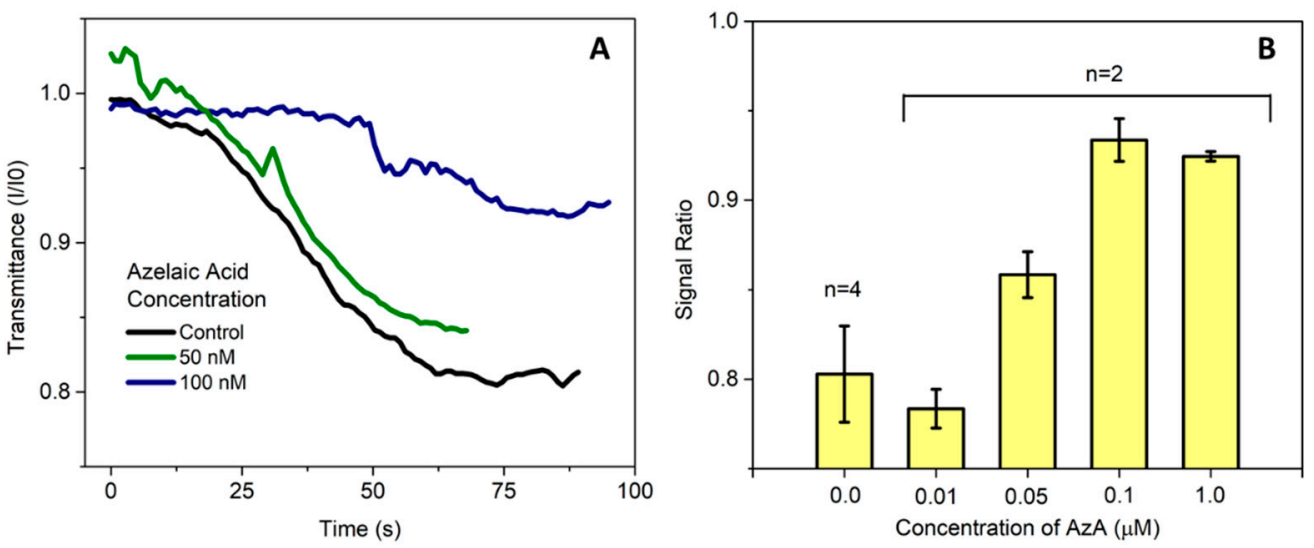

Figure 2. (A) Measurement of the microcolumn transmittance acquired in real time by the thin-film silicon photodiodes. The transmission values are presented as a ratio between the current at each time point and the initial value of measurement before introducing the sample; (B) Average value for endpoint measurements presented as the ratio between the final and initial current measured by the photodetector. Currently, the limit of detection of AzA in buffer in this system is approximately $50 \mathrm{nM}$.

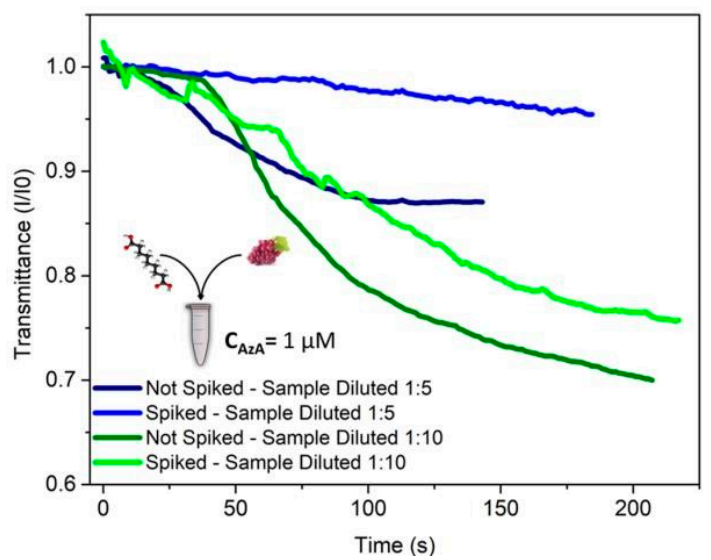

Figure 3. Detection of AzA in an artificially spiked sample of grape juice. The added concentration of AzA in the clarified grape juice was $1 \mu \mathrm{M}$. This mixture was then diluted in order to minimize the impact of matrix interferences from the grape juice itself. $35 \mu \mathrm{L}$ of sample was added to $15 \mu \mathrm{L}$ of a mixture of tyrosine and MBTH, resulting in final AzA concentrations of 140 and $70 \mathrm{nM}$. Measurements in raw grape juice was attempted (data not shown) but proved so far impossible to distinguish a contaminated from a non-contaminated sample due to the extent of the matrix interference. 


\section{Conclusions}

In this work, a microfluidic method for the detection of AzA in liquid samples is presented. The results obtained for buffer experiments allowed us to detect the organic acid at biologically relevant concentrations. To test the viability of the assay in a real scenario, AzA-spiked samples were prepared using fresh grape juice, where the detection of the molecule of interest was achieved. However, further development is necessary in order to improve sample pre-treatment and to integrate it on chip, with the goal of obtaining a true point-of-care device capable of being used in the field.

Author Contributions: Assay development and microfabrication were performed by E.J.S.B. A.M.F. and V.C. contributed to the troubleshooting of the biochemical assay and microfabrication, respectively. P.F. and J.P.C. supervised the study. All authors contributed to the writing of the manuscript.

Funding: E.J.S. Brás wishes to acknowledge FCT for the PhD grant PD/BD/128167/2016. The authors wish to thank FCT for funding through the research projects OptLOC (PTDC/BBB-NAN/5927/2014), and through Pluriannual financing of the Institute of Nanoscience and Nanotechnology (UID/NAN/50024/2013). In addition, the work was partially funded by the project POINT4PAC with funding from European Structural \& Investment Funds through the COMPETE Programme and from National Funds through FCT-Fundação para a Ciência e a Tecnologia under the Programme grant SAICTPAC/0019/2015.

Acknowledgments: The authors also wish to thank Denis Santos for his aid during the photo sensor fabrication process.

Conflicts of Interest: The authors declare no conflicts of interest.

\section{References}

1. Jung, H.W.; Tschaplinski, T.J.; Wang, L.; Glazebrook, J.; Greenberg, J.T. Priming in systemic plant immunity. Science 2009, 324, 89-91.

2. Schallreuter, K.U.; Wood, J.W. A possible mechanism of action for azelaic acid in the human epidermis. Arch. Dermatol. Res. 1990, 282, 168-171.

3. Santos, D.R.; Soares, R.R.G.; Chu, V.; Conde, J.P. Performance of hydrogenated amorphous silicon thin film photosensors at ultra-low light levels: Towards attomole sensitivities in labon-chip biosensing applications. IEEE Sens. J. 2017, 17, 6895-6903.

4. Pinto, I.F.; Soares, R.R.G.; Rosa, S.A.S.L.; Aires-Barros, M.R.; Chu, V.; Conde, J.P.; Azevedo, A.M. HighThroughput Nanoliter-Scale Analysis and Optimization of Multimodal Chromatography for the Capture of Monoclonal Antibodies. Anal. Chem. 2016, 88, 7959-7967.

5. Agudelo-Romero, P.; Erban, A.; Rego, C.; Carbonell-Bejerano, P.; Nascimento, T.; Sousa, L.; MartínezZapater, J.M.; Kopka, J.; Fortes, A.M. Transcriptome and metabolome reprogramming in Vitis vinifera cv. Trincadeira berries upon infection with Botrytis cinereal. J. Exp. Bot. 2015, 66, 1769-1785. 